\title{
Massive hemobilia following transpapillary bile duct biopsy treated by using a covered self-expandable metal stent
}

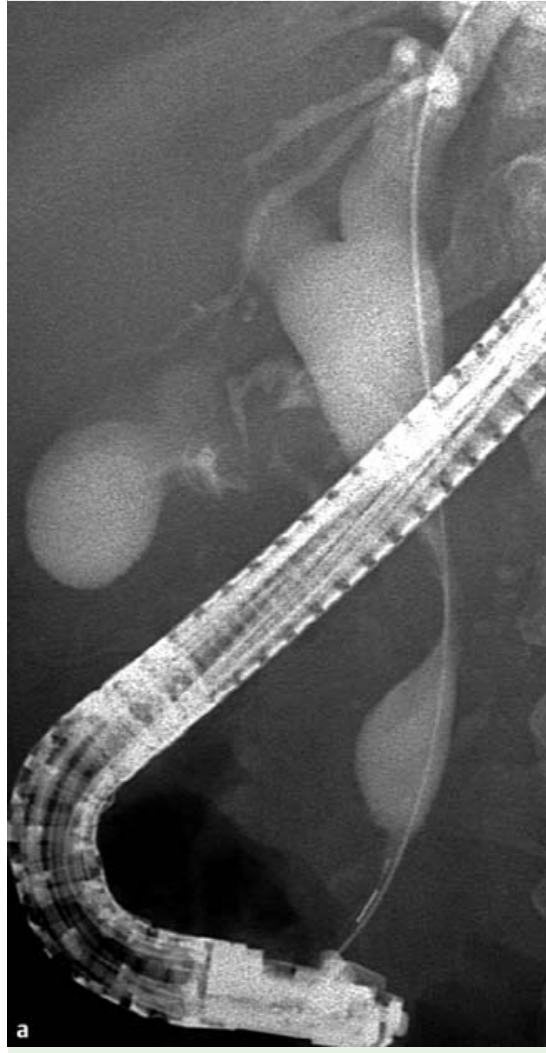

Fig. 1 a Cholangiogram showing luminal narrowing of the common bile duct with bile duct dilation. b Endoscopic transpapillary forceps biopsy was performed.

Severe hemobilia after a forceps biopsy is very rare $[1,2]$. The management of uncontrolled hemobilia after endoscopic procedures includes percutaneous radiologic intervention or surgery $[3,4]$. Recently, successful endoscopic hemostasis using covered self-expandable metal stents (CSEMS) for uncontrolled bleeding following sphincterotomy, stent removal, or papillary balloon dilation has been reported [3-5]. We report a case of massive hemobilia after a transpapillary forceps biopsy of the bile duct and successful endoscopic hemostasis with placement of a CSEMS.

A 51-year-old woman was admitted because of abdominal pain and jaundice. Abdominal CT showed cancer of the pancreatic head with a stricture of the common bile duct (CBD). Cholangiogram showed dilatation of the proximal bile

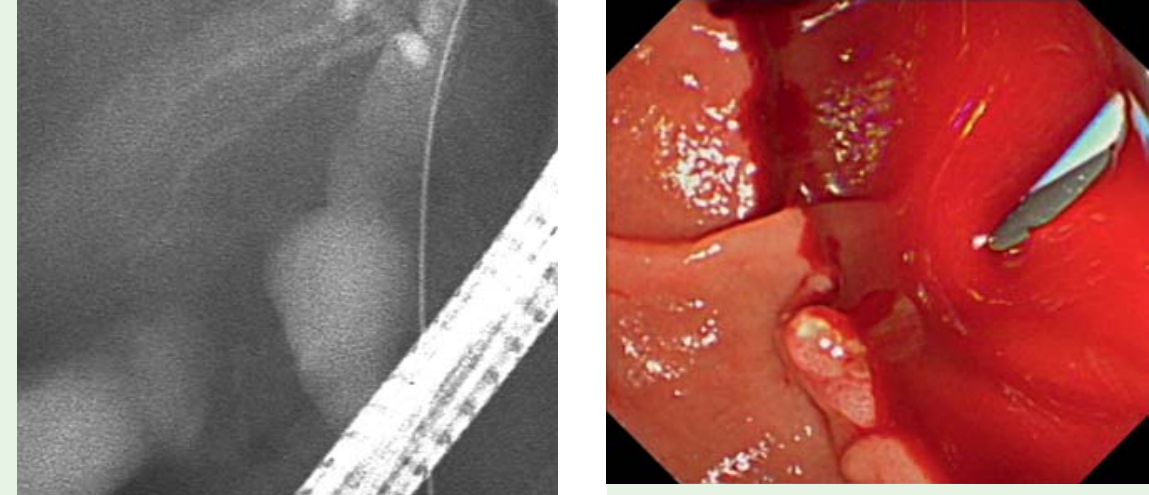

Fig.2 After the transpapillary forceps biopsy, massive hemobilia developed.

uncontrolled hemobilia continued, a partially covered SEMS (10 mm diameter, $8 \mathrm{~cm}$ long; Bonastent, Standard Sci-Tech, Seoul, Korea) was inserted to achieve hemostasis through compression exerted by the expandable stent ( Fig.3b). Once the CSEMS had been placed, the hemobilia decreased and then stopped. The patient's hemodynamic profile and vital signs were stable, and there was no further bleeding.

Complications related to transpapillary forceps biopsies are infrequent; usually, iatrogenic hemobilia is minor and can be controlled with conservative treatment [1]. Uncontrolled massive hemobilia after a transpapillary forceps biopsy has not previously been reported. Recently, placement of a CSEMS has been introduced as an effective means of endoscopic hemostasis for bleeding that could not be controlled with conventional methods [3-5]. If massive bleeding from the bile duct occurs during endoscopic retrograde cholangiopancreatography, hemostasis can be achieved promptly using a CSEMS, without radiological intervention or surgery. Endoscopic treatment using a CSEMS could be a useful way of providing effective hemostasis in selected patients with uncontrolled hemobilia of the extrahepatic bile duct.

\section{Video 1}

After a forceps biopsy was taken, sudden massive hemobilia occurred, flowing out of the duodenum. 


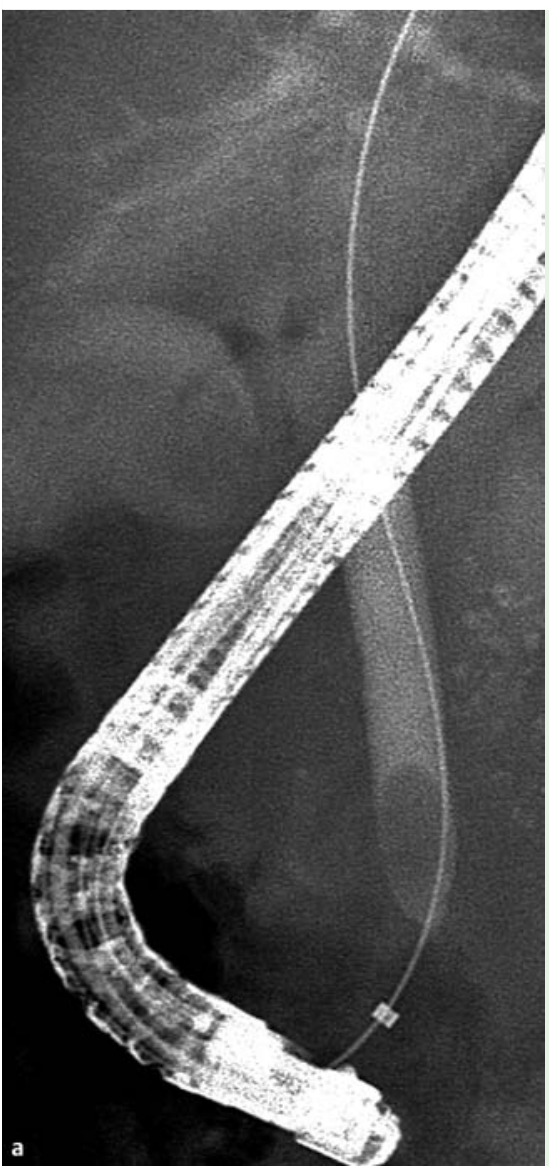

\section{Jeong-Yeop Song, Jong Ho Moon, Hyun Jong Choi, Dong Choon Kim, Moon Han Choi, Tae Hoon Lee, Sang-Woo Cha, Young Deok Cho, Sang-Heum Park}

Digestive Disease Center and Research Institute, Department of Internal Medicine, SoonChunHyang University School of Medicine, Bucheon and Seoul, Korea

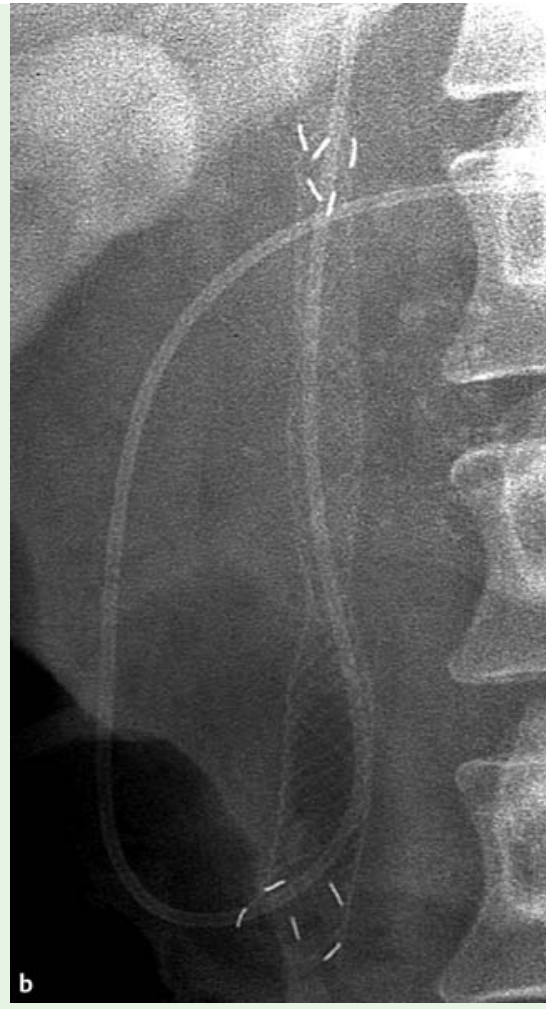

Fig.3 a Fluoroscopy showing balloon tamponade performed in an attempt to control the hemobilia. b A partially covered self-expanding metallic stent was placed with nasobiliary drainage.

\section{References}

1 Jailwala J, Fogel EL, Sherman $S$ et al. Tripletissue sampling at ERCP in malignant biliary obstruction. Gastrointest Endosc 2000; 51: $383-390$

2 Ikeda M, Maetani I, Terada Ket al. Usefulness of endoscopic retrograde biliary biopsy using large-capacity forceps for extrahepatic biliary strictures: a prospective randomized study. Endoscopy 2010; 42: 837-841
3 Itoi T, Yasuda I, Doi S et al. Endoscopic hemostasis using covered metallic stent placement for uncontrolled post-endoscopic sphincterotomy bleeding. Endoscopy 2011; 43: $369-372$

4 Valats JC, Funakoshi N, Bauret P et al. Covered self-expandable biliary stents for the treatment of bleeding after ERCP. Gastrointest Endosc 2013; 78: 183-187

5 Aslinia F, Hawkins L, Darwin P et al. Temporary placement of a fully covered metal stent to tamponade bleeding from endoscopic papillary balloon dilation. Gastrointest Endosc 2012; 76: 911 -913

\section{Bibliography}

DOI http://dx.doi.org/

10.1055/s-0034-1365115

Endoscopy 2014; 46: E161-E162

(c) Georg Thieme Verlag KG

Stuttgart · New York

ISSN 0013-726X

\section{Corresponding author}

\section{Jong Ho Moon, MD, PhD}

SoonChunHyang University School of Medicine Digestive Disease Center

SoonChunHyang University Bucheon Hospital 170 Jomaru-ro, Wonmi-gu Bucheon 420-767

Korea

Fax: +82-32-6215080

jhmoon@schmc.ac.kr 